\title{
A Minimally Constrained Optimization Algorithm for Table Cartograms
}

\author{
Andrew M. McNutt* Gordon Kindlmann \\ University of Chicago, Department of Computer Science
}

\begin{abstract}
Table cartograms are a recent type of data visualization that encodes numerical tabular data as a grid of quadrilaterals whose area are brought into correspondence with the input data. The overall effect is similar to that of a heat map that has been 'area-ed" rather than shaded. There exist several algorithms for creating these structuresvariously utilizing techniques such as computational geometry and numerical optimization - yet each of them impose aestheticallymotivated conditions that impede fine tuning or manipulation of the visual aesthetic of the output. In this work we contribute an optimization algorithm for creating table cartograms that is able to compute a variety of table cartograms layouts for a single dataset. We make our web-ready implementation available as table-cartogram.ts.
\end{abstract}

Index Terms: Human-centered computing-Visualization-Visualization techniques;

\section{INTRODUCTION}

Table cartograms are a specialized form of value-by-area maps, also called cartograms, that depict tabular data rather than geographic data [2]. They visualize a table of numbers as a grid of quadrilaterals, constrained to a single rectangle, whose areas are brought into correspondence with the input data. The visual effect is that of a shaded matrix that has been "area-ed" rather than merely colored. A given dataset can produce multiple equally accurate table cartograms because the problem is under-constrained [3].

Table cartograms were first studied by Evans et al. [2]. They demonstrate that every dataset admits a table cartogram by providing a constructive computational geometry algorithm. Subsequent work by Inoue and $\mathrm{Li}[3,4]$ found that it was more effective to generate them through mathematical optimization procedures. Unfortunately, both approaches are capable of generating only a single solution for a given dataset, which precludes the aesthetic control of the output or design-focused exploration of the solution space. Further, Inoue and Li's [3] approach relies on a MATLAB numerical solver, which is impractical for web-based applications, a common setting for contemporary visualizations.

In this work, we describe a method for creating table cartograms using an optimization approach that imposes a set of constraints that is equivalent to a minimal definition of the table cartogram. In particular we define them as a data visualization consisting of a planar grid of quadrilaterals constrained to a rectangle whose areas correspond to positive tabular input data. This broad and agnostic-to-aesthetic-desire definition allows us to produce table cartograms in a variety of aesthetics in the browser. We implement this algorithm as a typescript library, table-cartogram. $\mathrm{ts}^{1}$.

\section{Quantitative Description}

In order to execute an optimization approach we require a quantitative description of the table cartogram. We begin by requiring that

*e-mail: mcnutt@uchicago.edu

†e-mail: glk@uchicago.edu

${ }^{1}$ https://github.com/mcnuttandrew/table-cartogram

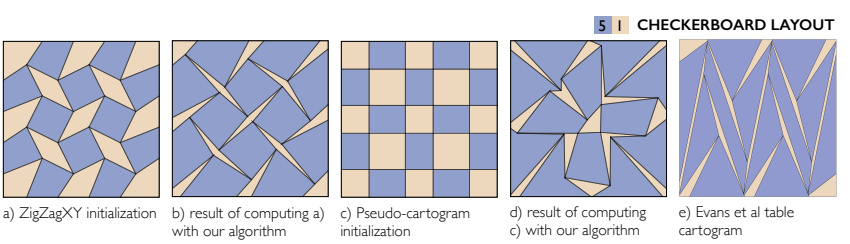

Figure 1: Table cartograms allow multiple outputs for the same input data. Here we show how different initializations can generate different outputs of equal accuracy.

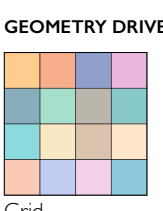

Grid

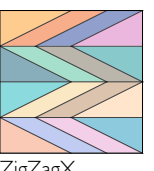

ZigZagX

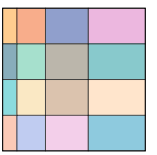

RampX

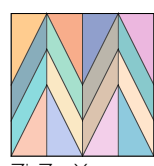

ZigZagY

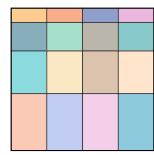

RampY

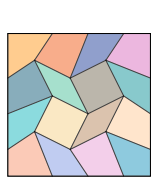

ZigZag $X Y$

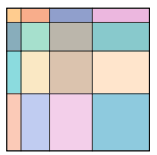

RampXY

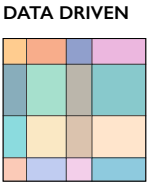

Pseudo-cartogram

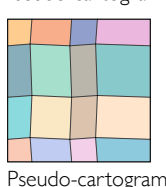

with ZigZag
Figure 2: Library of initializations for the our table cartogram method. The data driven layouts make use of Tobler's Pseudo-cartogram [7].

the visualization of the data is accurate (Accurate Data Embedding), which we express by demanding that each cell have the same ratio between its area and the area of the figure as its value and the sum of the values in the table. We formalize this as an error:

$$
\mathscr{E}=\frac{1}{\text { num cells }} \sum_{\text {cell } \in \text { table }} \frac{\left(\text { cell value }-\frac{\text { cell area }}{\text { table area }} \sum_{\text {value } \in \text { table }}\right)^{2}}{(\text { cell value })^{2}}
$$

A layout that accurately represents its data will have $\mathscr{E}=0$. The pixel nature of computer-based displays places an upper-bound on the precision of computed-based visualizations. While an ideal algorithm would have $\mathscr{E}=0$, it is sufficient to construct images that are correct by visual inspection, that is $\mathscr{E}<\varepsilon$.

Next, we require that the table cartogram follow a Planar Gridlike Topology. That is, the constituent quadrilaterals of the layout have exactly the expected number of neighbors, are non-intersecting, and are contained within the boundaries of a rectangle. We model this from a vertex perspective by demanding that the vertices must follow a consistent left-right and top-bottom ordering such that there is no overlap between the cells. There are a number of equally valid ways to define and enforce this property, for instance Inoue and Li [3] make use of the dual of our approach, optimizing from a quadrilateral-perspective. Our method uses a set of continuous measures that, in an accurate layout, will be zero. This aligns well with our definition as it must be the case that cells have value greater than zero, or else the topology of the layout would cease to be gridlike. Our quantitative definition closely follows previous discussions of accuracy in geographic cartograms $[1,5,6]$. 


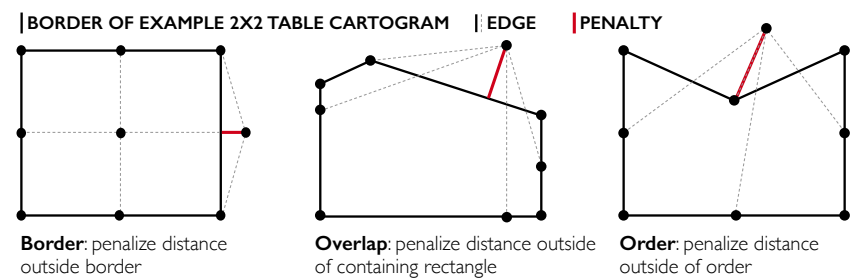

Figure 3: The constraints used to maintain the table cartogram topology. Each node is penalized by how much it violates the assertions.

\section{Optimization Approach}

We now describe our optimization-based approach. We sought an implementation that was easily applicable to the web and supported efficient exploration of the space of possible solutions, which we found to be unavailable under previous methods. We achieve these goals by applying a standard mini-batch stochastic gradient descent to an objective based on our quantitative description. In Fig. 1 we see that our approach achieves the accuracy of area embedding found in Evans et al. technique while offering a greater variability.

Our objective function consists of a loss function, which embeds data into the spatial layout (ensuring data accuracy), and a set of constraints modeling the table cartogram's geometry (ensuring planar topology). We use (1) as our loss. We maintain the desired topology through a trio of vertex constraints, which we describe textually below and diagrammatically in Fig. 3. We ensure that the vertices on the edges and corners of the table stay fixed by ignoring those vertices during the optimization process.

Border. Each vertex must remain within the border of the table cartogram. For a containing rectangle with upper left corner at $(0,0)$ and width $w$ and height $h$ we require that each vertex $v_{i}=\left(x_{i}, y_{i}\right)$ obey $0 \leq x_{i} \leq w$ and $0 \leq y_{i} \leq h$. For each coordinate we measure this as the maximum of the distance outside and zero.

Overlap. Each vertex must remain within a box defined by its up to eight neighbors. We model this constraint by giving a penalty equal to the minimum distance to an edge if the vertex is outside of the box and zero otherwise. This is a continuous analog of Tobler's algebraic sign maintenance [6].

Order. Each vertex must remain in both a monotonic $\mathrm{x}$ and a monotonic y ordering with its immediate neighbors. For each vertex component $v_{i, j}$, we require $v_{i-1, j}<v_{i, j}<v_{i+1, j}$ which we measure as the difference in expected order: $\max \left(v_{i+1, j}-v_{i, j}, 0\right)$. This prevents boxes from turning themselves inside out.

We apply these constraints via an exponential-hinge penalty, $f(x)=(|x|-x) e^{-x}$. This allows our solution to move freely between trust and penalty regions, while being pushed out of the penalty region. We combine these measures to form our constraint:

$$
\mathscr{C}(\vec{x})=\sum_{v_{i} \in \vec{x}} \lambda_{1} \operatorname{border}\left(v_{i}\right)+\lambda_{2} \operatorname{order}\left(v_{i}\right)+\lambda_{3} \operatorname{overlap}\left(v_{i}\right)
$$

Where $\lambda_{i}$ are implementation dependent scaling parameters. Our objective is then: $\operatorname{obj}(\vec{x})=\mathscr{E}(\vec{x})+\mathscr{C}(\vec{x})$. Both our constraints and our loss are continuous and differentiable, which enables us to use an analytic gradient of the objective.

We build our solutions using standard stochastic mini-batch gradient descent. Our choice of mini-batches takes advantage of the separable nature of the problem: each node in our lattice only contributes to the area of at most four quadrilaterals. As each quadrilateral consists of four vertices we are able to partition our vertices into four independent sets, which we reorder stochastically.

Our algorithm is able to construct a variety of different outputs for a single input dataset. To facilitate the control over the output we expose a variety of controls and optimization hyper-parameters such as step size and line search speed. The most expressive of these is the initialization state, as it confers geometric properties to the output. For instance, using an asymmetric initialization for a symmetric input data will result in a layout where the symmetry of the input data will be broken. Fig. 1 b) and d) shows two quite different variations of the same data. By default we attempt to intelligently select a good initialization by sweeping across a small library of layouts, Fig. 2, and selecting the one with the lowest objective value. This technique does not guarantee convergence and can require manual tuning of hyperparameters-such as the step size and layout initialization - to generate a result of desired accuracy and design.

\subsection{Analysis}

We now discuss the qualities of our algorithmic approach and its implementation. Our objective function and its gradient are linear with the size of the data: for a $n \times m$ input table the objective can be computed in $\mathscr{O}(\mathrm{nm})$. Despite this preferable complexity, the performance of our implementation is variable, likely due to both the irregularity of the problem space and the myopic nature of gradient descent. Our algorithm will sometimes sacrifice data accuracy for topological correctness-much in the same way as the closely related "Spatially Ordered Treemaps" [8] will sometimes exchange topological accuracy for data accuracy. This instability affects both the temporal performance of our system as well. For small, well-behaved tables (less than a few hundred cells whose range is less than a few orders of magnitude) we are typically able to find a solution very rapidly $(<1 s)$. Depending on the parameter tuning, a difficult table (e.g. one with a large range and non-smooth data) might take up to several minutes to arrive at a solution, or, as our solution is non-robust, may not converge at all. That said, we are mostly unconcerned with robustness - although future work might investigate it - as we merely sought an algorithm that could construct a variety of cartograms of sufficient accuracy for a given input. Our method—with careful tuning — our can do just that.

\section{Conclusion}

Table cartograms are a recent form of data visualization. We described a practical algorithm for constructing table cartograms using an optimization method that utilizes a minimal set of constraints. This allows us to create a variety of outputs that are accurate to a tunable accuracy, which is sufficient for visual application. Little is known about appropriate usage of the table cartogram. With our web-ready algorithm for their construction in hand, we intend to investigate to which applications table cartograms are best suited.

\section{ACKNOWLEDGMENTS}

The authors wish to thank Will Brackenbury and Mikita Vedzeneyeu for their helpful advice and commentary, as well as the reviewers.

\section{REFERENCES}

[1] M. J. Alam, S. G. Kobourov, and S. Veeramoni. Quantitative Measures for Cartogram Generation Techniques. In Computer Graphics Forum, vol. 34, pp. 351-360. Wiley Online Library, 2015.

[2] W. Evans, S. Felsner, M. Kaufmann, S. G. Kobourov, D. Mondal, R. I. Nishat, and K. Verbeek. Table cartogram. Computational Geometry, 68:174-185, 2018.

[3] R. Inoue and M. Li. Optimization-Based Construction of Quadrilateral Table Cartograms. ISPRS International Journal of Geo-Information, 9(1):43, 2020.

[4] M. Li and R. Inoue. Table Cartogram Generation as an Optimization Problem. Abstr. Int. Cartogr. Assoc, 1:1-2, 2019.

[5] S. Nusrat and S. Kobourov. The State of the Art in Cartograms. In Computer Graphics Forum, vol. 35, pp. 619-642, 2016.

[6] W. Tobler. Thirty five years of computer cartograms. ANNALS of the Association of American Geographers, 94(1):58-73, 2004.

[7] W. R. Tobler. Pseudo-cartograms. The American Cartographer, 1986.

[8] J. Wood and J. Dykes. Spatially Ordered Treemaps. IEEE Transactions on Visualization and Computer Graphics, 14(6), 2008. 